Miki Hiraoka • Fabio Rossi • Michael T. Trese

Barkur S. Shastry

\title{
X-linked juvenile retinoschisis: mutations at the retinoschisis and Norrie disease gene loci?
}

Received: October 2, 2000 / Accepted: November 7, 2000

\begin{abstract}
Juvenile retinoschisis (RS) and Norrie disease (ND) are X-linked recessive retinal disorders. Both disorders, in the majority of cases, are monogenic and are caused by mutations in the $R S$ and $N D$ genes, respectively. Here we report the identification of a family in which mutations in both the $R S$ and $N D$ genes are segregating with RS pathology. Although the mutations identified in this report were not functionally characterized with regard to their pathogenicity, it is likely that both of them are involved in RS pathology in the family analyzed. This suggests the complexity and digenic nature of monogenic human disorders in some cases. If this proves to be a widespread problem, it will complicate the strategies used to identify the genes involved in diseases and to develop methods for intervention.
\end{abstract}

Key words Retinoschisis $\cdot$ Norrie disease $\cdot$ Gene $\cdot$ Mutation $\cdot$ Deletion $\cdot \mathrm{X}$-linked

\section{Introduction}

X-linked retinoschisis (RS) is a bilateral vitreoretinal disease which results in poor visual acuity. It is characterized by intraretinal schisis and central and peripheral retinal lesions (Yanoff et al. 1968; George et al. 1996). The most typical clinical abnormality is foveal schisis, in $100 \%$ of eyes. Vitreous hemorrhage and retinal detachment are the most serious sight-threatening complications in advanced stages of the disorder. The majority of carriers do not show

M. Hiraoka $\cdot$ B.S. Shastry $(\varangle)$

Department of Biological Sciences, Oakland University, Rochester, MI 48309, USA

Tel. +1-248-370-2994; Fax +1-248-370-4225

e-mail: Barkur@aol.com

F. Rossi

Department of Molecular Pharmacology, Stanford University

Medical Center, Stanford, CA, USA

M.T. Trese

Department of Ophthalmology, William Beaumont Hospital, Royal Oak, MI, USA any ocular abnormality in the retina and cannot be identified clinically. The gene responsible for RS has recently been isolated (Sauver et al. 1997) and a spectrum of mutations has been described in affected families (den Dunnen 1998). Norrie disease (ND) is also a bilateral X-linked recessive syndrome, characterized by ocular dysgenesis, progressive mental retardation, and deafness. Bilateral blindness is frequently observed at birth (Warburg 1971). Histopathological studies reveal retinal detachment, large retinal vessels, falciform-fold formation, microophthalmia, and cataract (Warburg 1975). A full spectrum of mutations in the $N D$ gene have been identified in ND patients (Schuback et al. 1995; Shastry and Hiraoka 2000). In general, female carriers are completely healthy; however, cases in which female carriers manifest the disease have been described (Chen et al. 1993; Sims et al. 1997; Shastry et al. 1999). Both disorders, in most cases, are likely to be monogenic, although in some cases they exhibit a variable and overlapping clinical manifestation. This may pose diagnostic difficulties, and suggests the possibility of a polygenic nature of the disorders, but no such inheritance pattern has been described to date. Here we report the identification of a family in which mutations in both the $R S$ and $N D$ genes are segregating with RS pathology. These findings suggest that some cases of RS may be caused by two distinct genetic abnormalities.

\section{Subjects and methods}

Subjects

During our routine screening of families with retinoschisis and Norrie disease, we identified a unique four-generation family in which RS is segregating (Fig. 1). In this family, there is no male-to-male transmission, affected individuals are all males, and the transmission is from affected man to carrier daughter to affected grandson, all consistent with an $\mathrm{X}$-linked pattern of inheritance. A full-term male infant (IV-1 in Fig. 1) was born in 1997 to healthy parents, with an 


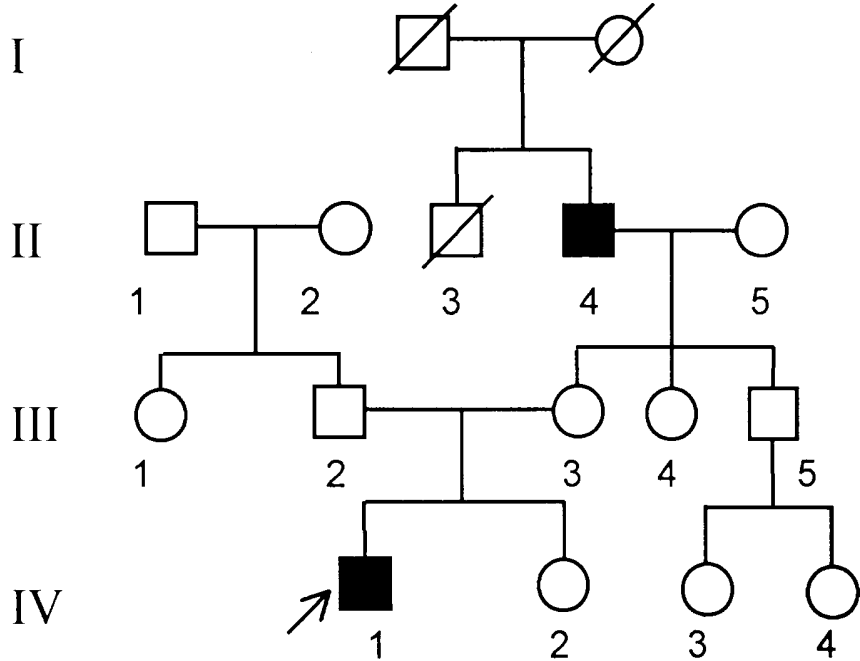

Fig. 1. Pedigree of a family in which retinoschisis (RS) is segregating. The clinically affected individuals are represented by the filled squares. Open symbols indicate unaffected individuals. Deceased individuals are marked with a slash. The proband is denoted by an arrow

uncomplicated delivery. The proband, when examined at the age of 2 years after birth, showed extensive schisis formation bilaterally, dragging of the discs, and hemorrhage. The child's maternal grandfather (II-4) also had bilateral retinal detachment, at the age of 40 years. However, no mental retardation and deafness have been noticed in either patient. The unaffected maternal grandmother (II-5), mother (III-3), maternal aunt (III-4) and uncle (III-5), and sister (IV-2) are phenotypically normal. Based on the characteristic fundus findings, the patients have been diagnosed as having a typical RS type pathology.

\section{Mutational analysis}

For the purpose of DNA analysis, leukocyte DNAs were extracted and all six exons of the $R S$ gene and three exons of the $N D$ gene were amplified by polymerase chain reaction (PCR), using commercially synthesized previously published primers (Sauver et al. 1997; Shastry et al. 1999) spanning the splice sites. The PCR products were purified and directly sequenced, using a cycle sequencing kit (Applied Biosystems, Foster City, CA, USA) according to the manufacturer's instructions. All samples were sequenced on both sense and antisense strands, using the same primers as for the PCR. The sequencing reactions were analyzed on $6 \%$ polyacrylamide gels containing $8 \mathrm{M}$ urea. The PCR amplifications were carried out by using Applied Biosystems Taq DNA polymerase, with 30 cycles of $1.5 \mathrm{~min}$ at $94^{\circ} \mathrm{C}, 1 \mathrm{~min}$ at $60^{\circ} \mathrm{C}$, and $2 \mathrm{~min}$ at $72^{\circ} \mathrm{C}$, in the manufacturer's buffer containing 2-4 units of polymerase, $10 \mathrm{pmol}$ each of the primer, $50 \mathrm{mM}$ each of four deoxynucleotides, $1.5 \mathrm{mM} \mathrm{MgCl}_{2}$, and $10 \mathrm{mM}$ tris- $\mathrm{HCl}, \mathrm{pH}$ 8.3. For restriction analysis, exon 4 of the $R S$ gene was amplified using the primers and genomic DNA as described above, but in the presence of $\alpha-{ }^{32} \mathrm{P}$ dATP. The amplified products were purified and digested with restriction enzyme
AluI (40 units) and the products were separated by $9 \%$ polyacrylamide gel electrophoresis.

Results

Although there are not too many reasons to search for mutations in the $N D$ gene in RS patients, we proceeded to analyze the $N D$ gene (an ongoing project in the laboratory), because it has recently been reported that mice carrying a disrupted $N D$ gene produced alterations in the murine eye which are similar to congenital retinoschisis (Reuther et al. 1997). The $N D$ gene contains three exons, and the protein coding sequence is confined within exons 2 and 3 , with the first exon untranslated. All three exons, including splice sites and the 3'-untranslated region, were sequenced (Meindl et al. 1992). Our extensive analyses revealed a 14bp deletion in the CT repeat region (exon 1) of the $N D$ gene in the affected proband (Fig. 2A), the unaffected maternal uncle (III-5), and the maternal grandfather (II-4). The unaffected mother, maternal grandmother, and maternal aunt (III-4) also carried the same deletion in one of their alleles; hence, this deletion mutation is segregating through three generations. Additionally, the unaffected father, the sister (IV-2), and 130 unrelated normal controls did not reveal a similar deletion. This deletion mutation in the family was further confirmed by single-strand conformational polymorphism (SSCP) analysis. To our knowledge, this mutation has not been previously reported, and, hence, represents a novel mutation in the $N D$ gene. Sequence analysis of exons 2 and 3 and the $3^{\prime}$ untranslated region failed to identify any additional mutations.

Because the maternal uncle, who carries the same deletion mutation, is asymptomatic, we next hypothesized that the pathology in this family required an additional gene defect at another locus. We found evidence strongly supporting our hypothesis when we screened this family for mutations in the $R S$ gene. The $R S$ gene contains six exons, and all six exons including splice sites were sequenced following PCR amplification, using the primers described before (Sauver et al. 1997). Sequence analysis identified a missense mutation (R102Q) in exon 4 of the $R S$ gene (Fig. $2 \mathrm{~B}$ ) in the proband and the maternal grandfather. The unaffected mother and maternal aunt (but not the unaffected father, maternal grandmother, maternal uncle, and sister) carried the same mutation in one of their alleles. This mutation was not found in 80 normal controls. The above sequence alteration changed the encoded amino acid arginine (CGG) to glutamine (CAG) and introduced a restriction site for the enzyme AluI. We have made use of this observation to verify the above sequencing data by freshly amplifying the genomic DNA and subjecting it to restriction analysis. The results are shown in Fig. 3. These data reconfirmed the above sequencing data in that, as expected, the maternal grandfather and the proband have the $R S$ gene mutation (lanes 2 and 6), whereas the maternal aunt and the mother are heterozygous for the same mutation (lanes 4 and 5). This mutation was not found in the father, maternal 
A

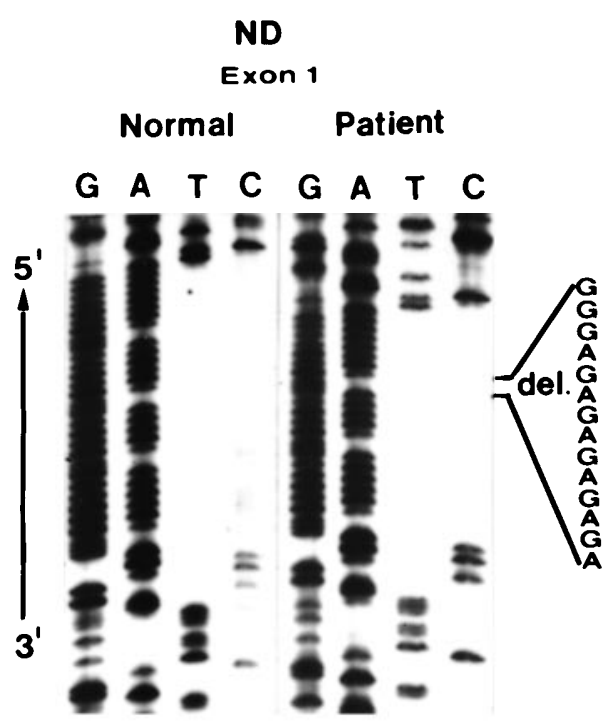

B

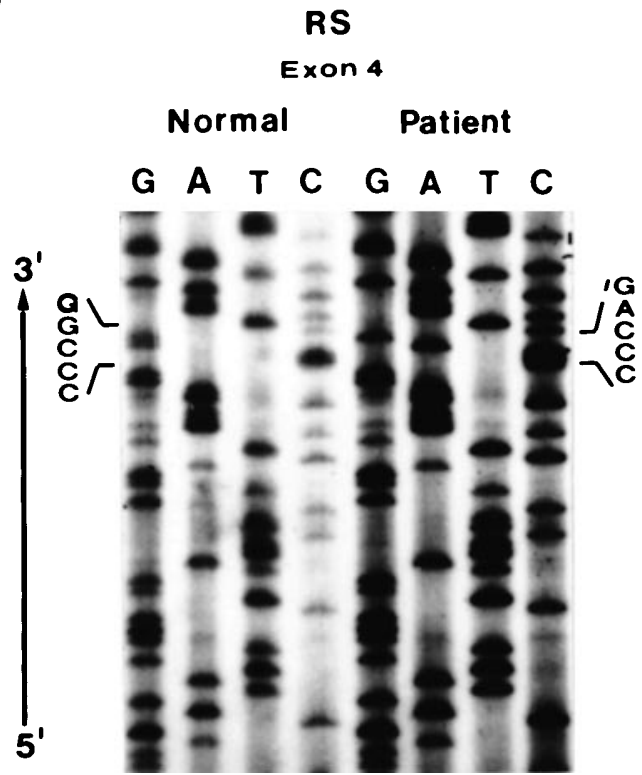

Fig. 2. A Nucleotide sequence of the mutant part of exon 1 of the $N D$ gene in individual IV-1 in the pedigree (Fig. 1). A 14-bp deletion is indicated on the right-hand side of Fig. 2A; this is also present in the maternal grandfather (II-4). The maternal grandmother (II-5), the mother (III-3) and the maternal aunt (III-4) carry the same deletion in one of their alleles. This mutation was not found in the unaffected father (III-2) or the sister (IV-2), or in 130 normal unrelated controls. Hence, the mutation is segregating with the disease through three generations. B Nucleotide sequence of the mutant part of the exon 4 of the $R S$ gene in individual IV-1. A missense mutation (CGG $\rightarrow$ CAG), which changed the amino acid arginine to glutamine, is segregating through three generations in the family. The mother and the maternal aunt carry the same mutation in one of their alleles. The maternal grandfather (but not the maternal grandmother, the father, or the sister) also carried the same mutation. This mutation was not found in 80 normal unrelated controls. This mutation has introduced a restriction site for the enzyme $A l u \mathrm{I}$

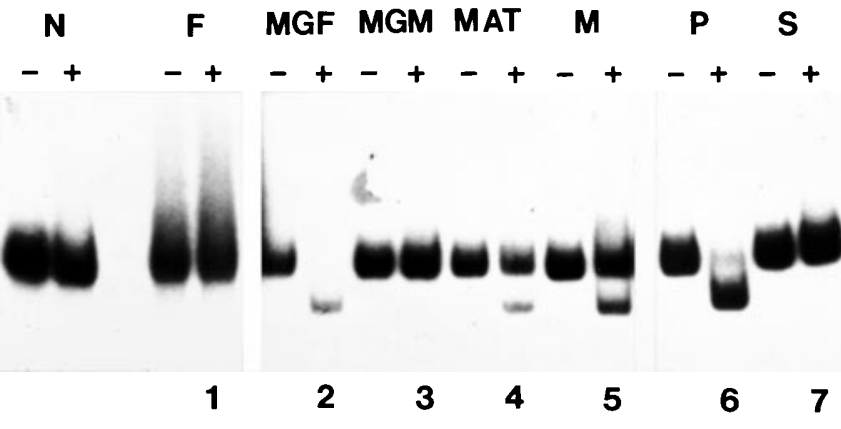

Fig. 3. Restriction digestion of the polymerase chain reaction (PCR) products to verify and show the segregation of the $R S$ gene mutation in the family. The presence of the digested PCR products in lanes 2, 4, 5, and 6 suggests the introduction of the $A l u \mathrm{I}$ site, which is not present in the normal control $(N)$, father (lane 1), maternal grandmother (lane 3), and sister (lane 7 ). The presence of an undigested fragment in addition to the digested products in lanes 4 and 5 indicate the carrier status of the maternal aunt and mother, respectively. The small fragment of the digested product is not shown. The designations + and - denote digestion with and without the AluI enzyme, respectively. $N$, Normal; $F$, father; $M G F$, maternal grandfather; $M G M$, maternal grandmother; $M A T$, maternal aunt; $M$, mother; $P$, proband; $S$, sister of the proband

grandmother, and sister (lanes 1, 3, and 7). Because this mutation has been reported previously by others (den Dunnen 1998) as a disease-causing mutation in other RS families, it is likely that this segregating mutation is also pathogenic in our family. Sequence analysis of the remaining exons failed to identify any additional mutations in the $R S$ gene. The above findings prompted us to examine an additional six RS families for mutations in the $N D$ gene, but we failed to identify additional families.

\section{Discussion}

The most interesting observation in this study is that of the digenic mutations in a single $\mathrm{X}$-chromosome in the family studied. The presence of the same deletion mutation in the $N D$ gene in both maternal grandparents argues for a consanguineous marriage in the family, but we were unable to confirm this fact with the available information. The contribution of each gene to the single phenotype in the family is also not clear at present. Both patients clinically exhibit a typical RS type pathology. This fact is, however, complicated by the finding that some of the ocular features, such as formation of the retrolental fibrovascular membrane, retinal traction, and retinal detachment are the common features of ND and RS which sometimes pose diagnostic difficulties (Godel and Goodman 1981). Because of this overlapping clinical presentation, the pathology contributed by each gene is perhaps not obvious clinically. It is also noteworthy that the affected maternal grandfather, who had mutations in both genes, did not have retinal detachment until his 40s, which is generally the case with $R S$ gene mutations (George et al. 1995, 1996). On the other hand, the affected proband showed retinal pathology at the age of 2 years. The reason for the difference in the age of onset is 
not clear at present. To the best of our knowledge, the great - grandparents (parents of the affected maternal grandfather) were unaffected. However, all affected individuals carried both the $R S$ and $N D$ gene mutations, suggesting the possibility that both genes are required for the pathology. The deletion mutation in the CT repeat region (promoter) may affect at the level of stability of the transcript or may cause lack of expression of the $N D$ gene (Kenyon and Craig 1999). Because mice lacking the $N D$ gene produce an RSlike pathology (Reuther et al. 1997), which could be caused by downregulation of the $R S$ gene (Lenzner et al. 2000), mutations in the $N D$ as well as the $R S$ genes in this family may result in a single RS pathology similar to digenic retinitis pigmentosa (Kajawara et al. 1994). One could argue that the maternal uncle carrying the $N D$ gene deletion alone is asymptomatic and that, therefore, the $N D$ gene mutation is polymorphic and is not pathogenic. Even in such cases, such CT repeat polymorphic mutation in the noncoding region could be one of the many predisposing factors for RS similar to that reported for rheumatoid arthritis (Khani-Hanjani et al. 2000). The fact that, (a) an identical deletion in the $N D$ gene has been found to be associated with a similar retinal pathology (retinal fold, retinal traction, retrolental fibrovascular membrane, and retinal detachment) in an unrelated patient (our unpublished results), (b) this deletion was not found in normal controls, and (c) the deletion in the CT repeat region has been shown to affect the expression of the gene (Kenyon and Craig 1999), it is very likely that it is pathogenic. However, because we do not have any affected or normal family members with only the $R S$ gene mutation, and expression assays with the mutated genes were not carried out, it is not possible to conclude whether the observed phenotype is indeed caused by the mutations identified in this report.

Whatever the explanation of the mechanisms of pathogenesis, examples of digenic mutations in human diseases are very rare. The other digenic autosomal diseases which have been described in the literature are retinitis pigmentosa, Waardenberg syndrome type 2, and AntleyBixler syndrome (Kajawara et al. 1994; Morell et al. 1997; Reardon et al. 2000). In retinitis pigmentosa, the phenotype is due to mutations in two different genes at two different chromosomes. In the present family, both $R S$ and $N D$ are $\mathrm{X}$-chromosomal genes, and the phenotype in the proband is likely to be due to segregating mutations in these two genes. These results suggest that in some cases, $\mathrm{X}$-linked monogenic disorders can have digenic mutations. This may explain the wide phenotypic expression of the disorders even within a single family. If this proves to be a wide-spread problem, it will complicate the strategies used to identify the genes involved in diseases, the use of DNA technology to detect the carriers, and the methods developed for intervention with existing procedures. In such cases, several genes will have to be screened, using the candidate gene approach, to obtain some kind of confidence for using DNA as a diagnostic test.

Acknowledgments We thank the family members who kindly donated blood samples for the study. This work was supported by a grant from the Retinopathy of Prematurity Foundation (ROPARD).

\section{References}

Chen Z-Y, Bettinelli EM, Woodruff G, Young I, Breakefield XO, Craig IW (1993) Characterization of mutation within the NDP gene in a family with a manifesting female carrier. Hum Mol Genet 2:1727-1729

den Dunnen JT (1998) The retinoschisis consortium. Functional implications of the spectrum of mutations found in 234 cases with $\mathrm{X}$ linked juvenile retinoschisis. Hum Mol Genet 7:1185-1192

George NDL, Yates JRW, Moore AT (1995) X-linked retinoschisis. Br J Ophthalmol 79:697-702

George NDL, Yates JRW, Moore AT (1996) Clinical features in affected males with X-linked retinoschisis. Arch Ophthalmol 114:274280

Godel V, Goodman RM (1981) X-linked recessive primary retinal dysplasia: clinical findings in affected males and carrier females. Clin Genet 20:260-266

Kajawara K, Berson EL, Dryja TP (1994) Digenic retinitis pigmentosa due to mutations at the unlinked peripherin/RDS and ROM1 loci. Science 264:1604-1608

Kenyon JR, Craig IW (1999) Analysis of the 5' regulatory region of the human Norrie disease gene: evidence that a non-translated CT dinucleotide repeat in exon one has a role in controlling expression. Gene 227:181-188

Khani-Hanjani A, Lacaille D, Hoar D, Chalmers A, Horsman D, Anderson M, Balshaw R, Keown PA (2000) Association between dinucleotide repeat in non-coding region of interferon - gamma gene and susceptibility to, and severity of, rheumatoid arthritis. Lancet 356:820-825

Lenzner S, Prietz S, Feil S, Schulz R, Ropers H-H, Berger W (2000) Analysis of gene expression in a mouse model for Norrie disease by cDNA subtraction and microarrays. Am J Hum Genet 67 (Suppl):381

Meindl A, Berger W, Meitinger T, van de Pol D, Achatz H, Dorner C, Haasemann M, Hellebrand H, Gal A, Cremers F, Ropers H-H (1992) Norrie disease is caused by mutations in an extracellular protein resembling C-terminal globular domain of mucin. Nat Genet 2:139-143

Morell R, Spritz RA, Ho L, Pierpont J, Guo W, Friedman TB, Asher JH Jr (1997) Apparent digenic inheritance of Waardenberg syndrome type 2 (WS2) and autosomal recessive ocular albinism. Hum Mol Genet 6:659-664

Reardon W, Smith A, Honour JW, Hindmarsh P, Das D, Rumsby G, Nelson I, Malcolm S, Ades L, Sillence D, Kumar D, DeLozierBlanchet C, McKee S, Kelly T, McKechan WL, Baraitser M, Winter RM (2000) Evidence for digenic inheritance in some cases of AntleyBixler syndrome. J Med Genet 37:26-32

Reuther K, Van de Pool D, Jaissel G, Berger W, Tarnow RP, Zrenner E (1997) Retinoschisis like alterations in the mouse eye caused by gene targeting of Norrie disease gene. Invest Ophthalmol Vis Sci 38:710-718

Sauver CG, Gehrig A, Warneke-Wittstock R, Marquardt A, Ewing CC, Gibson A, Lorenz B, Jurklies B, Weber BHW (1997) Positional cloning of the gene associated with $\mathrm{X}$-linked juvenile retinoschisis. Nat Genet 17:164-170

Schuback DE, Chen Z-Y, Craig IW, Breakefield XO, Sims KB (1995) Mutations in the Norrie disease gene. Hum Mutat 5:285-292

Shastry BS, Hiraoka M, Trese DC, Trese MT (1999) Norrie disease and exudative vitreoretinopathy in families with affected female carriers. Eur J Ophthalmol 9:238-242

Shastry BS, Hiraoka M (2000) Molecular genetics of familial exudative vitreoretinopathy and Norrie disease. Curr Genomics 1:259-269

Sims KB, Irvine AR, Good MV (1997) Norrie disease in a family with a manifesting female carrier. Arch Ophthalmol 115:517-519

Warburg M (1971) Norrie disease. Birth defects 7:117-124

Warburg M (1975) Norrie disease. Differential diagnosis and treatment. Acta Ophthalmol 53:217-236

Yanoff M, Rahn EK, Zimmerman LE (1968) Histopathology of juvenile retinoschisis. Arch Ophthalmol 79:49-53 